\title{
Deep brain stimulation in Parkinson's disease: The selection of appropriate patient candidate
}

\author{
Bekir Enes Demiryurek \\ Department of Neurology, Acıbadem Healthcare Group Clinic, Kocaeli, Turkey
}

\begin{abstract}
A BSTRACT
Deep brain stimulation surgery, one of the device-assisted treatments for advanced Parkinson's disease, provides a significant improvement in quality of life criteria when applied to the appropriate candidates. Although the subthalamic nucleus is the main neuroanatomical structure for this surgery, other surgical targets include thalamus ventral intermediate (VIM) and globus pallidus internus. Candidates are selected and evaluated by a dedicated team for deep brain stimulation surgery. The deep brain stimulation team consists of a neurosurgeon, a psychiatrist, a neuroradiologist, an anesthesiologist, and a neurologist, who specialize in movement disorder. The patient's motor symptom response to levodopa treatment and other contraindications are reviewed, and the patient is prepared for deep brain stimulation surgery. This article addresses the criteria for the selection of patients with advanced Parkinson's disease, eligible for deep brain stimulation treatment, as well as addressing the appropriate anatomical targets.
\end{abstract}

Key words: Parkinson's disease (PD), deep brain stimulation (DBS), appropriate candidates, therapy.

$\triangle$ Dr. Bekir Enes Demiryurek

Department of Neurology, Acıbadem Healthcare Group Clinic, Kocaeli, Turkey

E-mail: bekirenes10@gmail.com

Received: 2021-05-07 / Accepted: 2021-05-18

Published online: 2021-06-01

\section{Introduction}

In recent years, the device-assisted treatment options are applied for the treatment of patients with advanced Parkinson's disease (PD). The definition of advanced stage patient has recently been discussed and re-defined. Patients with severe dyskinesia or severe off periods, patients with at least two hours of off time during the day, postural instability during the off period, painful dystonia, and severe freezing during the off — regardless of the duration of the disease - are eligible for device-assisted treatment [1-5]. However, the most important assessment scale, except for the situations defined, is the restricted daily life activities. Device-assisted treatments include apomorphine pump-continuous infusion, duodopa intestinal infusion, and deep brain stimulation (DBS) [1-5].

\section{Patient selection}

The cause of a third of ineffective surgery outcomes in Parkinson's disease is "wrong candidate selection" [6]. In candidate selection, the patient is referred to surgery by taking into account the risk/benefit ratio. Patient-specific findings and surgical risks of patients with Parkinson's disease are evaluated by a team of experts. The deep brain stimulation team consists of a neurosurgeon, a psychiatrist, a neuroradiologist, an anesthesiologist, and a neurologist, who specialize in movement 
disorder. In some centers, the team also includes an internal medicine specialist, neurophysiologist, and Parkinson's disease nurse. The ideal patient for deep brain stimulation is a patient with early onset, a good levodopa response, and uncontrollable motor complications. However, some symptoms of patients may have partial or no response to levodopa [7]. The most important factor to be considered in patient selection is the planning of how to control the levodopa unresponsive symptoms after surgery.

For planning, the following questions need to be answered:

1. Is there an age limit?

2. Are there surgical contraindications?

3. Is there a cognitive dysfunction?

4. If there are too many Levodoparesistant symptoms (dysarthria, dysphasia, postural instability and walking-balance problems), what will be done and how will the patient be followed up after surgery?

\section{Age}

Age is an independent variable, but surgery is not recommended for patients over 75 years of age, although there is no age limit. However, the physiological age of the patient is always taken into account more than his/her calendar age. Looking at the studies, we can see that about a quarter of subthalamic nucleus (STN) and globus pallidus internus (GPi) DBS cases are over the age of 70 . Considering the risk/benefit ratio, cognitive reserve in older patients is more limited, levodopa-resistant symptoms are more frequent, life expectancy is shorter, and there are more accompanying diseases [8].

\section{Surgical contraindications}

Uncontrolled hypertension before surgery increases the risk of intracerebral hematoma 10fold during surgery [8]. Moreover, uncontrolled heart disease, permanent stroke and active infection increases the surgical risks. Preoperative magnetic resonance (MRI) examinations are used to rule out structural lesions that increase risk, and small vessel disease and Parkinson-plus syndromes that affect utilization and invite behavioral problems after surgery. Magnetic resonance findings are not appropriate in 9\% of patients selected in the clinic, and these patients are excluded from surgical treatment. Moreover, patients with severe cortical atrophy also have a high risk of subdural hematoma after surgery. Other factors that the neurologist should check for and pay attention to when preparing the patient for surgery are as follows:

1. Skin infections

2. Use of drugs effective on bleeding

3. Risk of psychosis and confusion during or after surgery

4. Ability to switch from high-dose and multi-drug treatment to a simpler treatment scheme:

For dopamine agonists and rasagiline, ability to reduce the dose weeks ago and simplify treatment.

Advanced stage patients should be informed about the expected benefits, possible risks, and technical issues and usage problems of all treatments.

\section{Cognitive dysfunction}

Dementia is the definitive exclusion criterion for the deep brain stimulation treatment. In these patients, executive functions are severely affected. Patients with advanced age and borderline cognitive function experience an increase in frontal and executive function losses, and decrease in verbal fluency, especially after STN DBS. It is known that 
these findings are due to the lesion effect, and do not improve by turning off the stimulator or adjusting the voltage. Identifying patients at risk by evaluating cognitive functions with standard tests is of importance for the success of the treatment. After determining the neuropsychological profile of the patient, psychiatry is consulted to evaluate severe depression, suicide attempts or psychotic symptoms independent of medications experienced in the preoperative period, in addition, the family history of severe psychiatric illness is questioned. Some patients are excluded at this stage. Especially in the first year after surgery, the risk of suicide increases in cases of STN DBS due to the rapid discontinuation of dopaminergic drugs [8]. Patients with Parkinson's disease whose history includes suicide attempts and whose disease started at a young age are not suitable candidates for STN DBS.

\section{Levodopa response}

The most important criterion affecting treatment success in surgical treatment is the response of the patient's symptoms to levodopa. Especially for STN DBS, the expected motor benefit in the postoperative period is similar to the levodopa response. If the patient's signs of resistance to levodopa are intense and these symptoms are responsible for the main disability, these patients are not suitable for surgical treatment. When evaluating the Levodopa response, the best on periods of the patient are evaluated, and if necessary, an extra dose is given to see the best on periods of the patient. Patients who are active after Levodopa (at least 1 hour) are the best candidates for STN DBS [9].

In the advanced stage Parkinson's disease, the severity and type of dyskinesia are not important when referring the patient to surgical treatment since both STN and GPi DBS treatments control dyskinesia. However, STN DBS requires a slow voltage increase in the early postoperative period, while in GPi DBS, the patient quickly recovers from dyskinesia after surgery.

There is no defined severity of the disease that will change the way patients benefit from surgical treatment. Dramatic improvements can be seen in patients with high motor scores. Disability and personal factors that the patient is exposed to due to Parkinsonian symptoms determine the success of surgical treatment.

The disease duration is not a primary factor in patient selection for surgical treatment. Looking at patients selected for subthalamic nucleus DBS, it seems that the average duration of disease is 7.5 years, and the average duration of motor complications is 3 years. As the duration of the disease increases, the risk of surgical implantation, technical problems $(17.2 \%)$ and intracranial bleeding (1-2\%) increases $[8,10]$. Therefore, if the patient has impaired quality of life data despite an optimal treatment, the patient can be referred to surgical treatment, provided that the diagnosis of the disease is accurate. Surgical treatment is usually planned after the first five years, except for patients with resistant resting tremor.

\section{Determination of the surgical target and follow-up of the patient in the postoperative period}

Thalamus VIM DBS is selected as a target in patients with resting tremor resistant to levodopa and other treatments in Parkinson's disease. Dysarthria and balance problems may occur in patients due to bilateral stimulation. Another group of patients include patients who are not suitable for other targets due to their age and cognitive profiles: In these patients, severe tremor is easily controlled by unilateral 
thalamic stimulation. It is ineffective for bradykinesia and rigidity.

Subthalamic nucleus DBS is the most selected practice. It is effective against bradykinesia, rigidity and tremor. Some of the side-effects associated with bilateral stimulation can be overcome by programming. Dyskinesia, paresthesia and dysphonia occur in $19 \%$ of patients, but these are reversible. In $41 \%$ of patients, it slows down memory, executive functions and mental speed. These patients should be monitored for depression and hypomania. Weight gain and balance problems occur in almost every patient. Its main advantage is that it reduces the dose of dopaminergic drugs [11-5].

Globus pallidus internus DBS is effective in all cardinal findings, but makes a dramatic reduction in dyskinesia. Adverse-effect profile is lower than STN DBS. The dose of dopaminergic drugs does not change in the post-operative period.

In deep brain stimulation surgery, early postoperative complications include intracerebral hemorrhage (2\%), ischemic stroke $(1 \%)$, seizure $(0-3 \%)$, and postoperative confusion (21\%); and, implant site infection (3$8 \%$ ) is seen in follow-ups [8]. Especially after STN DBS, cognitive decline, impaired frontal executive functions, decreased verbal fluency and impaired working memory are seen [7].

Neuromodulation settings are performed every 1-2 weeks in the first months of the patient's postoperative follow-up. The average optimal setting time varies from patient to patient in the range of 1-3 months. In subsequent follow-ups, the patient is called to the clinic to check the impedance and parameters at intervals of six months.

\section{Conclusion}

As one of the device-assisted treatments for advanced Parkinson's disease, DBS provides a significant improvement in quality of life criteria when applied to the appropriate patient. STN is the preferred target, but thalamus VIM and GPi are other surgical targets. The most important criterion determining the patient's response to surgery is the levodopa response of the patient's findings. Especially after STN DBS, the patient's antiparkinsonian drug doses can be reduced, so the patient is also protected against the drug side-effects. After DBS surgery, which is applied to the appropriate patient at the appropriate time with the appropriate neuroanatomic target, the followup of patients also requires care and patience.

Funding: The author(s) received no financial support for the research, authorship, and/or publication of this article.

Conflict of Interest: The authors declare that they have no conflict of interest.

Ethical statement: Since this research is a review study, no ethics committee decision was required.

\section{Open Access Statement}

This is an open access journal which means that all content is freely available without charge to the user or his/her institution under the terms of the Creative Commons Attribution NonCommercial License (http://creativecommons.org/licenses/bync/4.0). Users are allowed to read, download, copy, distribute, print, search, or link to the full texts of the articles, without asking prior permission from the publisher or the author.

\section{References}

[1]Malek N. Deep Brain Stimulation in Parkinson's Disease. Neurol India. 2019;67(4):968-78.

[2]Dayal V, Limousin P, Foltynie T. 
Subthalamic Nucleus Deep Brain Stimulation in Parkinson's Disease: The Effect of Varying Stimulation Parameters. J Parkinsons Dis. 2017;7(2):235-45.

[3]Umemura A, Oyama G, Shimo Y, et al. Deep brain stimulation for Parkinson's disease. Nihon Rinsho. 2017;75(1):83-88.

[4]Mohammed A, Bayford R, Demosthenous A. Toward adaptive deep brain stimulation in Parkinson's disease: a review. Neurodegener Dis Manag. 2018;8(2):115136.

[5]Janssen ML, Duits AA, Turaihi AH, et al. Subthalamic nucleus high-frequency stimulation for advanced Parkinson's disease: motor and neuropsychological outcome after 10 years. Stereotact Funct Neurosurg. 2014;92(6):381-87.

[6]Rodriguez-Oroz MC, Rodriguez M, Guridi $\mathrm{J}$, et al. The subthalamic nucleus in Parkinson's disease: somatotopic organization and physiological characteristics. Brain. 2001;124(Pt 9):177790.

[7]Volkmann J, Albanese A, Kulisevsky J, et al. Long-term effects of pallidal or subthalamic deep brain stimulation on quality of life in Parkinson's disease. Mov Disord. 2009;24(8):1154-61.

[8]Kleiner-Fisman G, Herzog J, Fisman DN, et al. Subthalamic nucleus deep brain stimulation: summary and meta-analysis of outcomes. Mov Disord. 2006; Suppl 14:S290-304.

[9]Welter ML, Houeto JL, Bonnet AM, et al. Effects of high-frequency stimulation on subthalamic neuronal activity in parkinsonian patients. Arch Neurol. 2004;61(1): 89-96.

[10] Agnesi F, Connolly AT, Baker KB, et al. Deep brain stimulation imposes complex informational lesions. PLoS One. 2013;8(8):e74462.

[11]Rizzone MG, Fasano A, Daniele A, et al. Long-term outcome of sub- thalamic nucleus DBS in Parkinson's disease: from the advanced phase towards the late stage of the disease? Parkinsonism Relat Disord. 2014;20(4):376-81. 